\title{
Parametric CAD Modelling of Aircraft Wings for FEA Vibration Analysis
}

\author{
Shengyong Zhang*, Mike Mikulich \\ Department of Mechanical and Civil Engineering, College of Engineering and Sciences, Purdue University Northwest, Westville, USA \\ Email: *syzhang@pnw.edu
}

How to cite this paper: Zhang, S.Y. and Mikulich, M. (2021) Parametric CAD Modelling of Aircraft Wings for FEA Vibration Analysis. Journal of Applied Mathematics and Physics, 9, 889-900.

https://doi.org/10.4236/jamp.2021.95060

Received: March 31, 2021

Accepted: May 14, 2021

Published: May 17, 2021

Copyright $\odot 2021$ by author(s) and Scientific Research Publishing Inc. This work is licensed under the Creative Commons Attribution International License (CC BY 4.0).

http://creativecommons.org/licenses/by/4.0/

(c) (i) Open Access

\begin{abstract}
Excessive vibration of aircraft wings during flight is harmful and may cause propagation of existing cracks in the material, leading to catastrophic failures as a result of material fatigue. This study investigates the variations of modal characteristics of aircraft wings with respect to changes in the structural configurations. We develop parametric Computer-Aided Design (CAD) models to capture new design intend on the aircraft wing architectures. Subsequent Finite Element Analysis (FEA) based vibration analysis is performed to study the effects of architecture changes on the wing's natural frequencies and mode shapes. It is concluded that the spar placement and the number of ribs have significant influence on the wing's natural vibration properties. Integrating CAD modelling and FEA vibration analysis enables designers to develop alternative wing architectures to implement design requirements in the preliminary design stage.
\end{abstract}

\section{Keywords}

Parametric Modelling, CAD, Aircraft Wings, FEA, Vibration Analysis

\section{Introduction}

The primary function of aircraft wings is to generate the required lifting force during taking-off and flight. Wings are also made to store the jet fuel, mount the turbine engines, and store the retractable landing gear systems in some designs. In its most basic form, an aircraft wing is comprised of spars, ribs, and outer panels. Spars are the main load-carrying elements and extend from the fuselage to the tip through the wing. Ribs are structural elements attached to the spars and span between the leading edge to the trailing edge. Ribs are designed to provide required airfoil shape and transmit the external loads to the wing spars. The outside skin covers the entire surface of the wing. Wings are subjected to a com- 
bination of the propulsive force, drag force, lifting force and gravitational force in all phases during flight. The vibration of aircraft wings due to these exciting forces may cause propagation of existing cracks in the material, leading to catastrophic failures as a result of material fatigue. It is essential to consider the vibration characteristics in the design of wings to satisfy the desired stability and academic performance.

Much research has been reported on the computer simulation of aircraft wings for structure optimizations. CAD and FEA based computer simulations play a crucial role in the design, evaluation, and modification of aircraft wings. There are many wing design variables, and the simulation results help designers evaluate "what if" scenarios. Rao [1] conducted FEA analysis for a wing with specified dimensions and material properties to optimize the cross sections of the spars and ribs when the wing was subjected to a combination of flight load, fuel load, and engine load. Topology optimization was carried out for the same wing after replacing the aluminum alloys with composites. Modal analysis was performed in this work to study the transient responses of the wing due to an impact of a bird. Kuntoji and Kuppas [2] developed a wing CAD model for studying the vibration responses of the wing subjected to aerodynamic excitations. The first ten natural frequencies and corresponding vibration modes of this specific wing were acquired in this study. Kavya G. and Reddy R. [3] conducted FEA-based analysis to investigate the mechanical behavior of an A320 commercial airplane wing, which constitutes two spars and multiple ribs. Except of the stress analysis for the wing structure, modal analysis was performed in this work to study the effects of containing the spars and ribs within the wing on the changes of its natural frequencies and corresponding mode shapes. Zhu and Childs [4] reviewed the development of lightweight design approaches in the airframe components, especially the light weighting approaches applied in the design of solar powered aircraft wings. Different lightweight materials, including aluminum alloys, titanium alloys, aerospace composites, were compared on their properties as well as the advantages each material has. A number of computer-aided structural optimization methods (for example, sizing optimization, shape optimization and topology optimization) and an array of advanced manufacturing techniques (for example, foam metal, advanced metal forming techniques, etc.) were reviewed with their applications in the aircraft wing designs.

In this paper, we build parametric CAD models to capture the design intent for modifying wing architectures. We choose four user-defined design variables for the parametric CAD models in this research, including the wing length, wing tape ratio, spar location, and the number of ribs in a wing. Any changes in these variables will yield a change in the wing configurations and a corresponding updating of the CAD model. Subsequent FEA-based vibration analysis of the resulting wing models is performed for studying the effects of changes in these design variables on the vibration modal characteristics in forms of natural frequencies and mode shapes [5]. 


\section{Parametric CAD Models of Wings}

Among many wing design variables, we choose the wing length, spar placement, number of ribs and the taper ratio as the four user-defined variables for our parametric CAD models in this study. The wing length defines the length of a wing, measured from the side that attaches to the fuselage to the wing tip. The taper ratio is the ratio of the lengths of two chords at the root and tip side, respectively. The spar placement allows one to change the position of the spar inside the wing. Its value ranges from 0 to 1 . For example, a value of 0.25 will place the spar one-quarter of the chord length from the leading edge, a value of 0.5 will place the spar one-half of the chord length from the leading edge, a value of 0.75 will place the spar three-quarter of the chord length from the leading edge, and so forth. The number of ribs specifies how many ribs the wing has. By default, the ribs are evenly distributed between the root and the tip of the wing. Any changes in the values of these design variables will yield a change in the wing configurations. The effects of the architecture changes on the vibration responses of the wings can be studied in the subsequent FEA analysis.

The modelling process begins by preparing the airfoil profile coordinates in a Microsoft Excel spreadsheet. In this research, we utilize the data points for an Althaus AH 79-100A airfoil as the baseline profile [6]. The airfoil profile is first created at the root side of the wing by importing the data from the spreadsheet to Autodesk Inventor programming. The import process establishes points at the coordinates but does not set dimensions. This means that even though the points are displayed at their proper locations, there are no constraints to keep them at that location. The point on the leading edge is constrained to always be at the origin of the coordinate system, and every other point is constrained with dimensions referencing this origin. Since the imported profile coordinates are defined for a chord with unit length, one can easily change the actual dimensions of airfoil profiles by changing the desired chord length. When all data points are fully constrained, spline functions are used to create a smooth curve that goes through each of the defined locations to create the airfoil profile geometry. Due to the abrupt changes in geometry on the trailing edge, we generate two separate spline functions to model the top side and the bottom side, respectively. Both spline functions start from the point on the leading edge and end at the point on the trailing edge through each point between them sequentially. In order to create a model that incorporates the user-defined taper ratio, we cannot simply extrude the sketch that we have just created. Instead, we have to create a second sketch at the tip of the wing. To make this sketch, a new workplane is established offset from the plane of the first sketch. The distance of this offset equals to the length of the wing, which is one of the four design variables. On the present workplane, the same coordinates are imported again, and the process of constraining the sketch is repeated with minor modifications. In order to keep the wing tip centered with the root, the point on the leading edge could not be set to coincide with the origin. The constraint to locate the leading edge at the 
wing tip has to be a function incorporating the root chord length and tip chord length simultaneously. All of the other points can then reference this point in the same fashion as before. Once the second sketch is completed, a surface loft can be generated to define the geometry of the outer covering of the wing.

A single wing spar is modelled in this study. The spar is modeled by creating a new sketch on each workplane previously established for the root and tip. The thickness of the spar member is defined in the modelling processing as well. The location of the spar is determined by the input value to the user-design variable for placing the spar. The value of the parameter ranges from 0 to1, allowing one to place the spar anywhere between the leading edge and the trailing edge. The spar is then built by connecting the sketches at the tip and root with a loft to match the shape of the wing.

The number of ribs is a user-defined variable too, so they could not be sketched out in the same manner as the root and tip are. Allowing the user to select the number of ribs changes the location of the ribs inside the wing, in addition to a user defined taper ratio, resulting in unique cross-sections for each rib. This eliminates the possibility of modeling one rib and then copying it a number of times according to the user input. To create a universal rib model, a rectangular surface is created that references the root chord length, which must always be greater than the tip chord length in this study. This surface is defined to extend beyond the wing skin at any point on the wing and the excess can be trimmed later. With a surface defined that can locate a rib at any point, we can copy this sketch according to the user input. A rectangular array is created for the surface that would reference the wing length and number of ribs, which are both design variables in this study, in order to create evenly spaced ribs throughout the wing.

A parametric CAD model is complete at this point. In order to use the model, one can load the Inventor file on the workstation. A model of the wing will be present, with specified values assigned to the user input variables. One can change the values of these variables displayed in the variable dialogue box and the CAD model will update its configuration subsequently to capture new design intent. Figures 1-4 show the changes in the configurations of a wing with respect to the user-defined variables.
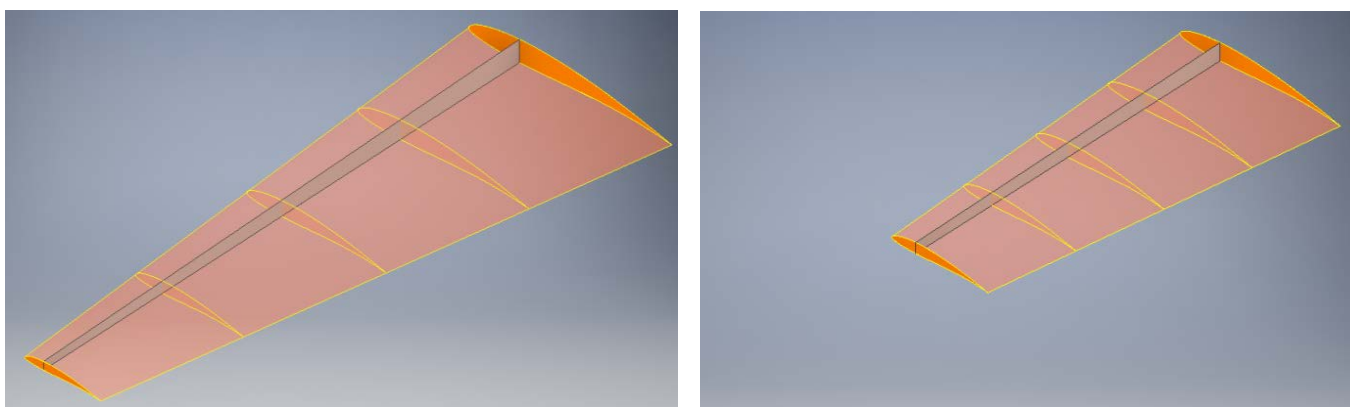

Figure 1. Changes in the wing configurations with respect to changes in the design variable of wing length. 

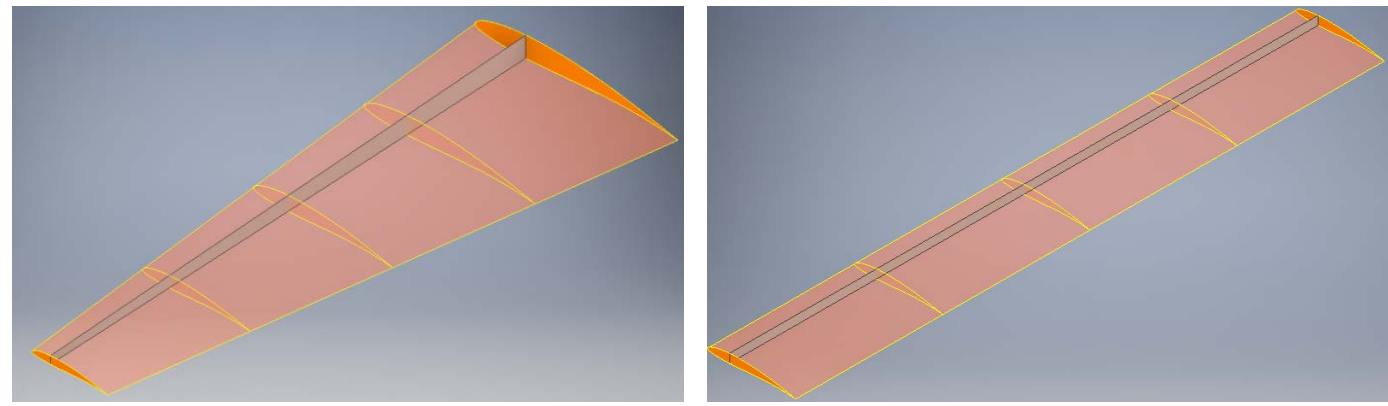

Figure 2. Changes in the wing configurations with respect to changes in the design variable of wing taper ratio.
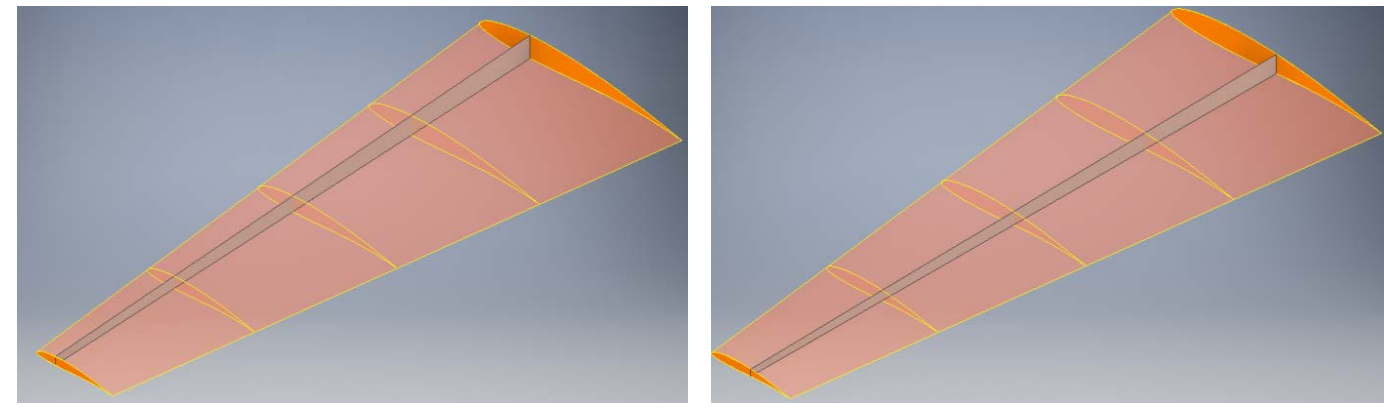

Figure 3. Changes in the wing configurations with respect to changes in the design variable of spar location.
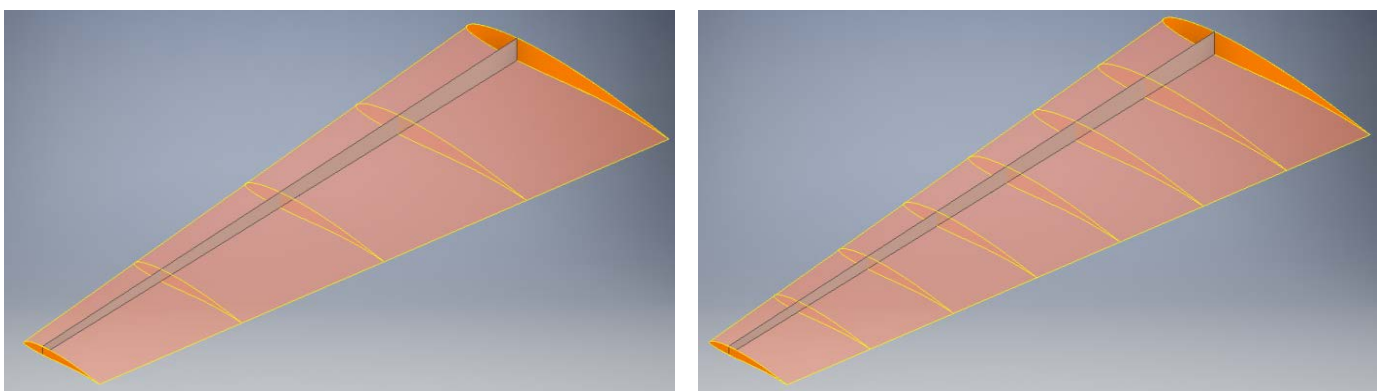

Figure 4. Changes in the wing configurations with respect to changes in the design variable of number of ribs.

\section{FEA Vibration Analysis of Wings}

Structural FEA analysis has been widely applied to simulate the behaviors of a system under operating conditions and to evaluate different designs of the system in the preliminary design stage. One of the most attractive characteristics in using CAD and FEA based computer simulations is that designers can change the values of design variables and observe corresponding changes in the system responses. Besides, the visual features (animation, contour plot, for example) enable designers to visualize the inherent properties of a structure.

Once the wing CAD model has been completed, we can export the CAD model as an IGES file and import the IGES file to ANSYS for vibration analysis. Before converting the CAD model to FEA model, a few additional modifications need to be made to simulate the rigid connections between the spar and each rib, the spar and outer skin, and the outer skin and each rib. Each rib is to be divided 
into two parts by the spar: one is between the leading edge and the spar and the other is between the spar and trailing edge. The spar is to be divided into multiple segments, one segment representing the part in between two adjacent ribs. Both the top and bottom outer panels are to be divided into multiple quadrilaterals as well, as shown in Figure 5. These minor modifications ensure the rigid connections among the spar, outer panels, and ribs in the resulting FEA model after discretization. As an example, Figure 6 shows all the finite elements are properly attached at the nodes between the spar and rib.

At this point, it is ready to build FEA model for vibrational analysis. We choose shell elements to mesh the wing structure. One can define different material properties and thicknesses for meshing the spar, ribs, and outer skins. It is common to simulate the behavior of a fixed wing as a cantilever beam, with the wing supported at the fuselage. With the built FEA model, we are able to conduct a series of vibration analyses for the user-defined wing to learn the changes of its responses to changes in the design variables under different excitations, including the free responses and forced responses. Free responses of the wing result from nonzero initial displacement and/or velocity excitations and provide an insight of the inherent damping property of the wing. Forced responses to

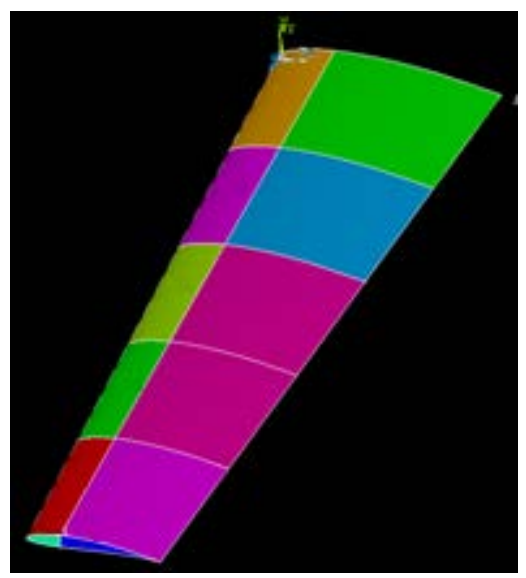

Figure 5. The top and bottom outer panels are divided into a number of quadrilaterals.

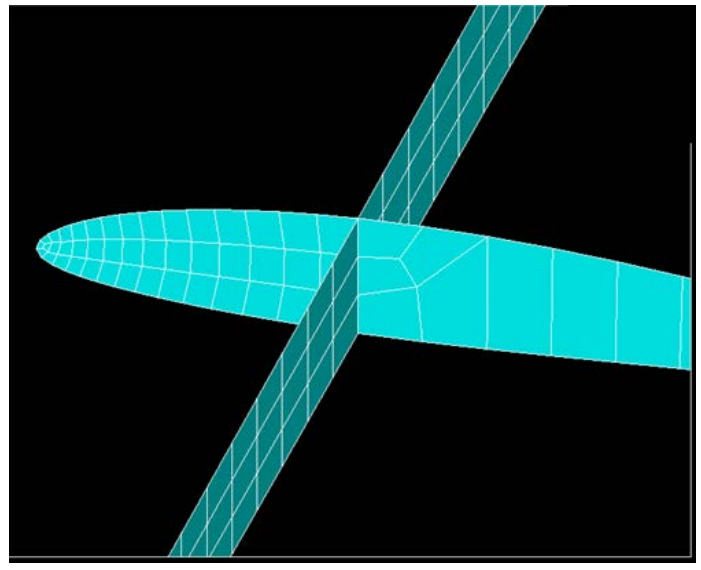

Figure 6. Rigid connections between the spar and ribs are created in the FE model. 
harmonic excitations provide a means to study the resonant behavior of the wing. When the frequency of a periodic external force is nearly the same as one natural frequency of the wing on which it acts, the resulting vibration displacement may be very large and exceed the elastic limits that the wing can sustain. Impulsive response of the wing is the result of a shock loading (for example, the wing hits a guest of wind) and can be further used for studying vibration responses to general force excitations based on convolution integrals.

In this paper, we focus on the modal analysis based on the FE models. Modal analysis is a technique to identify the inherent properties of a vibrating system in terms of natural frequencies and mode shapes. A vibration mode is a natural state of vibration with a displacement configuration corresponding to a specific natural frequency. In this study, we conduct modal analysis by constraining all the degrees of freedom of each node at the wing root. Figure 7 shows the FEA

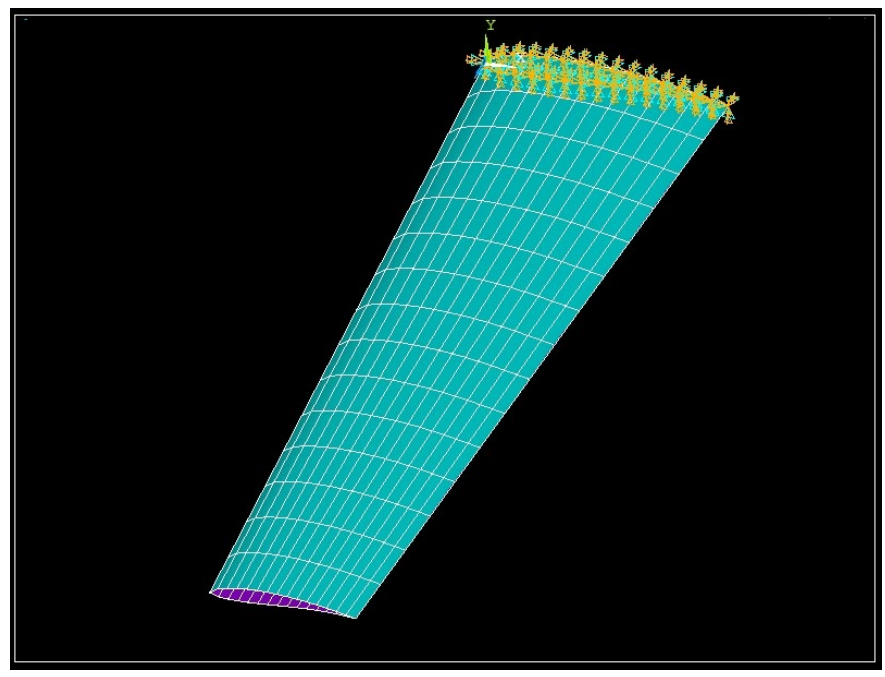

(a)

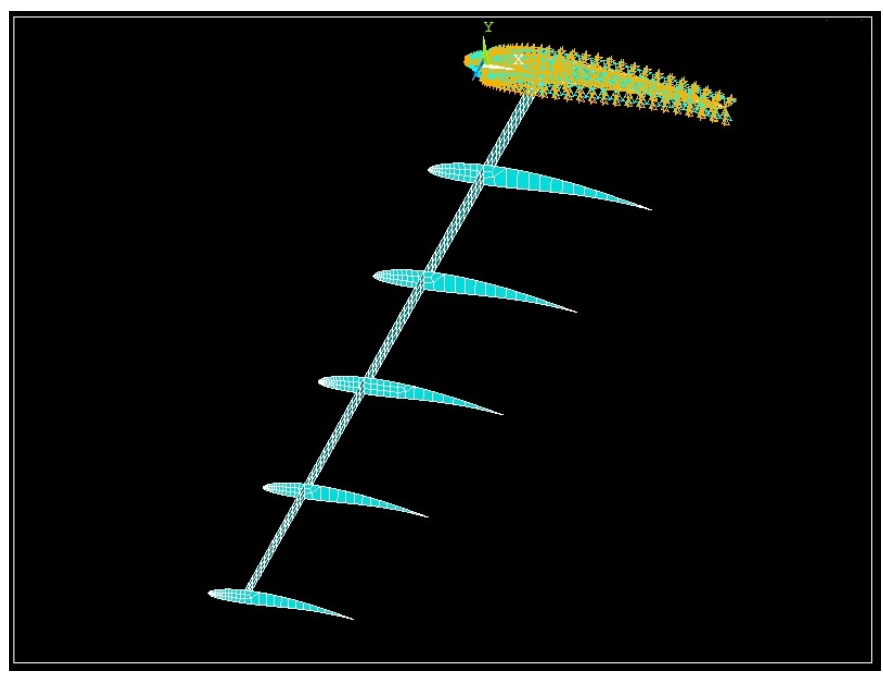

(b)

Figure 7. FEA models of a user-defined wing for vibration modal analysis. (a) The outer skin and (b) the spar and ribs after discretization. 
models of the wing. To capture new design intent, one can change the wing configurations by assigning new values to the design variables in the above construction of parametric CAD models. The resulting CAD model is in turn imported to ANSYS for studying the effects of these changes on the vibration properties of the wing.

Without losing generality, we focused on the first three natural frequencies and vibration modes in our discussion. Figure 8 shows the first three vibration

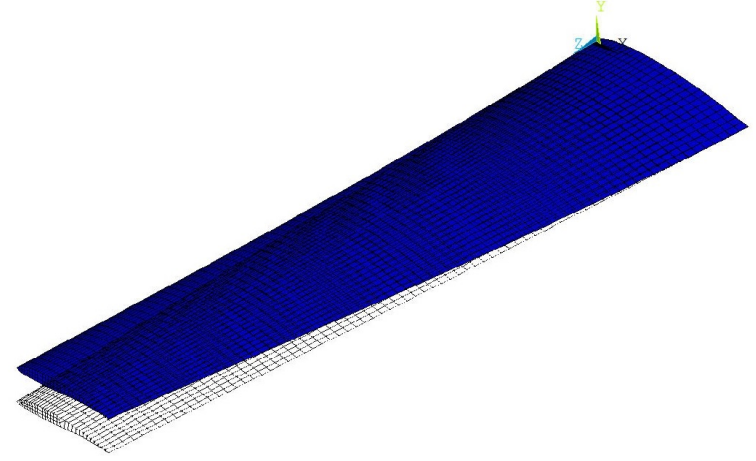

(a)

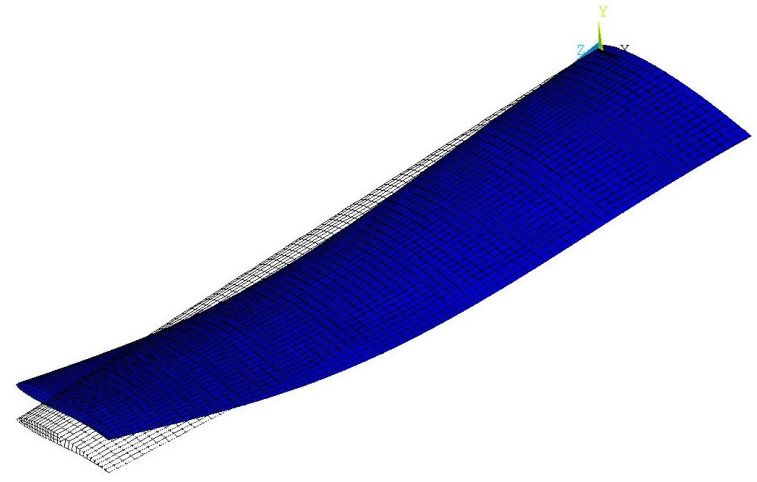

(b)

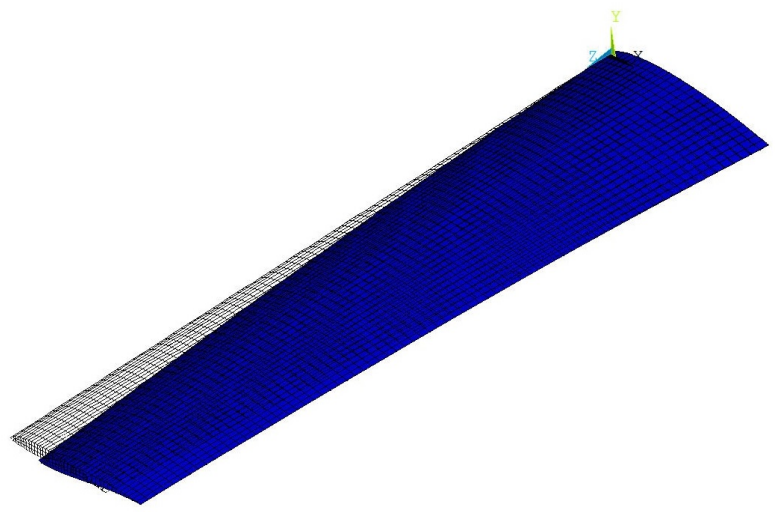

(c)

Figure 8. Vibration modes of a wing with 7 ribs and the spar is 0.4 times of the chord length from the leading edge. (a) The fundamental bending vibration mode, (b) the second order bending vibration mode, and (c) the fundamental torsional vibration mode. 
modes in which the first two are bending vibration modes and the third one a torsional vibration mode. Figure 9 shows the variations of the three natural frequencies with respect to changes of the spar placement in the wing $(0.2,0.4,0.6$ and 0.8 times of the chord length from the leading edge, respectively). It is obvious that changing the spar location has different effects on the bending and

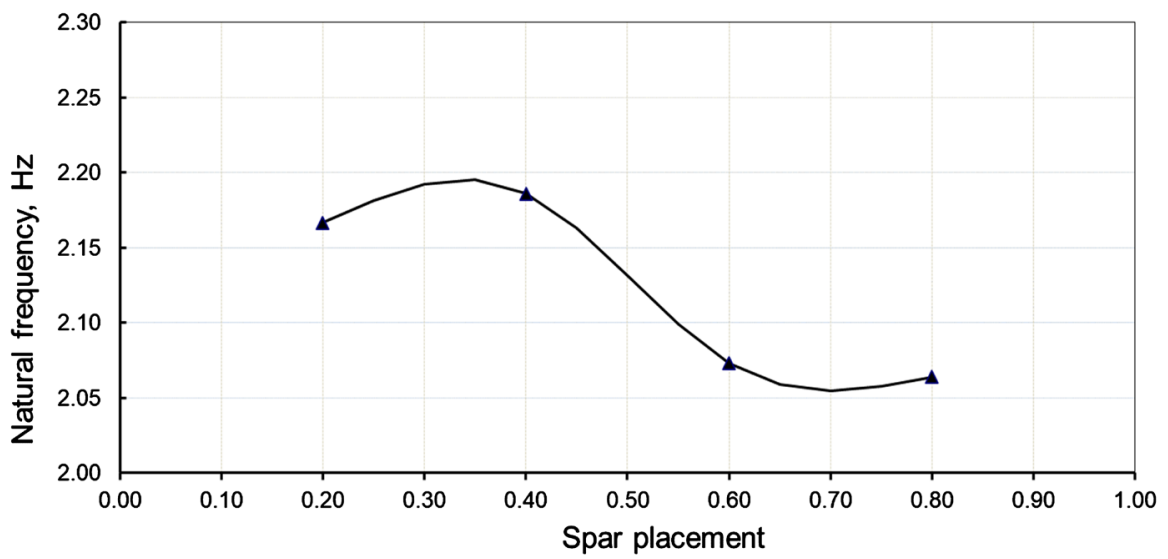

(a)

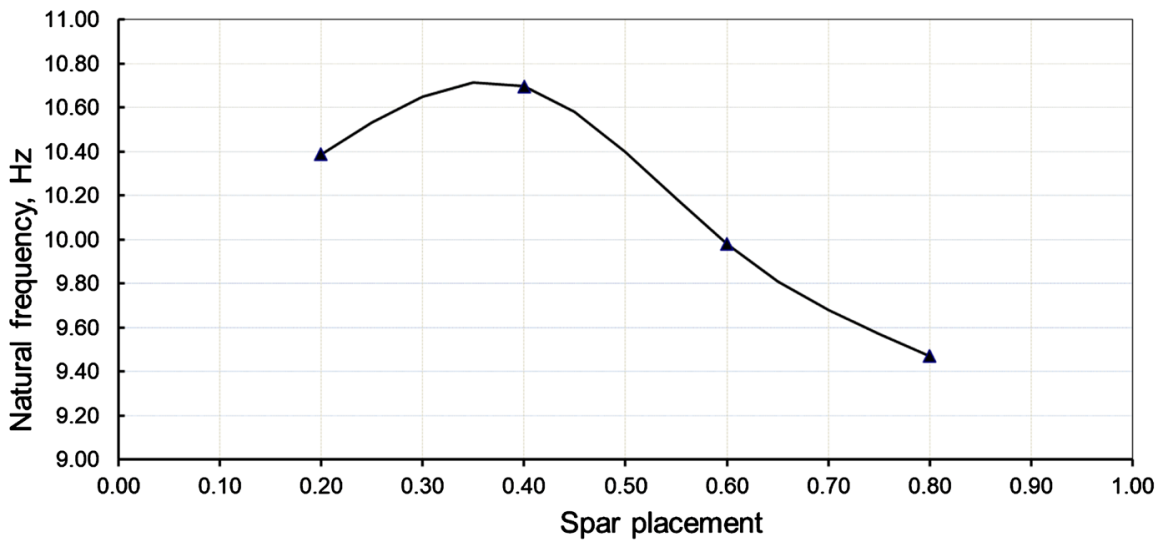

(b)

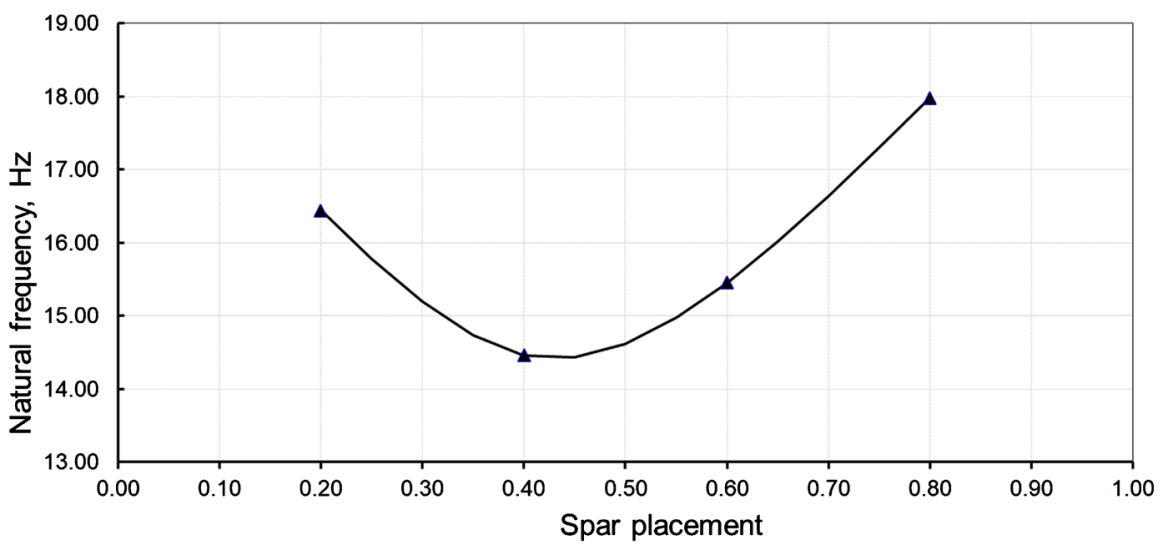

(c)

Figure 9. Variations of (a) the fundamental bending natural frequency, (b) second order bending natural frequency and (c) fundamental torsional natural frequency with respect to spar locations. 
torsional natural frequencies. Moving the spar member to the central portion of the wing helps improve the bending natural frequencies but results in a decrease in the torsional natural frequencies. This can be interpreted as a decrease in the torsional stiffness of the wing structure. Figure 10 shows the variations of the

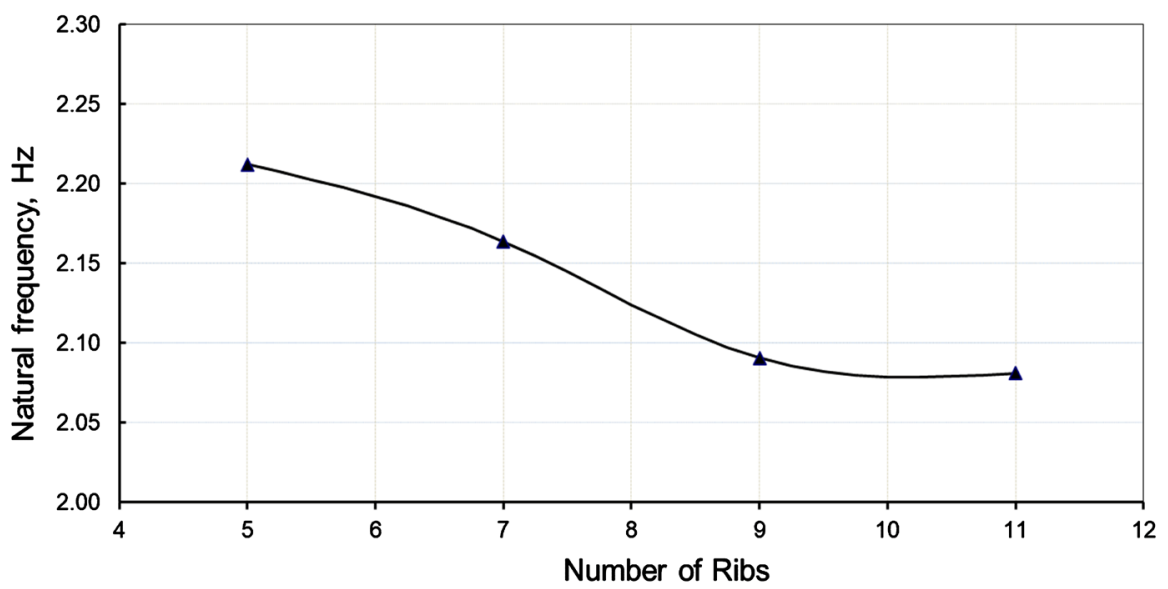

(a)

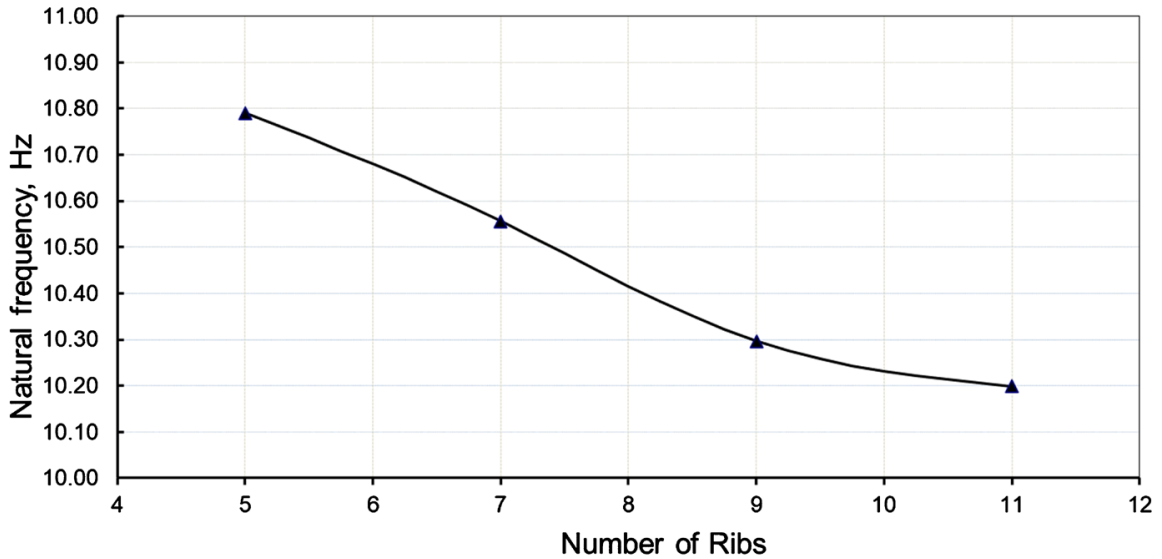

(b)

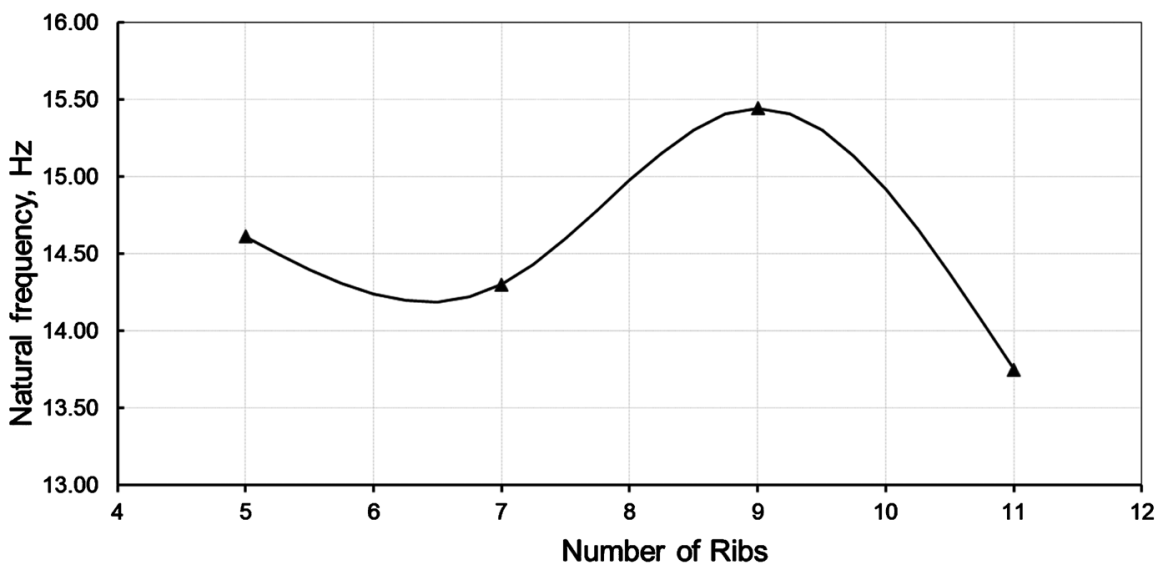

(c)

Figure 10. Variations of (a) the fundamental bending natural frequency, (b) second order bending natural frequency and (c) fundamental torsional natural frequency with respect to the number of ribs. 
three natural frequencies with respect to the number of ribs $(5,7,9$ and 11, respectively). It is found that increasing the number of ribs of the studied wing model has an inverse influence on the bending natural frequencies. The maximum torsional natural frequency of this wing model is achieved by employing nine ribs in the design. It is clear that parametric CAD models allow designers to capture the design intent for modifying wing architectures. Any changes in the wing length, tape ratio, spar location and number of ribs in this study will yield a change in the wing configurations, and the wing CAD model will update itself automatically to accommodate the new designs. The subsequent FEA-based modal analysis will reveal the corresponding inherent dynamic characteristics of the wings in forms of natural frequencies and vibration modes. It is concluded that integrating $\mathrm{CAD}$ modelling and FEA vibration analysis enables designers to develop alternative wing architectures to implement design requirements in the preliminary design stage.

\section{Conclusion}

Integrating $\mathrm{CAD}$ modelling and FEA vibration analysis enables designers to develop alternative wing architectures to implement design requirements in the preliminary design stage. Parametric CAD models created in this research allow one to easily model any rectangular or trapezoidal wings with a single spar. Both the number of ribs and the spar placement can be altered according to new design considerations and requirements. In conjunction with FEA vibration analysis, the inherent dynamic characteristics of user-defined wings have been analyzed in forms of natural frequencies and mode shapes. It is concluded that both the spar placement and the number of ribs within a wing have significant influence on the wing's natural vibration properties.

\section{Acknowledgements}

This research was sponsored through the Indiana Space Grant Consortium (INSGC). The authors are grateful to the personnel in this organization for the funding.

\section{Conflicts of Interest}

The authors declare no conflicts of interest regarding the publication of this paper.

\section{References}

[1] Rao, J.S. (2016) Advances in Aero Structures. Procedia Engineering, 144, 3-25.

[2] Kuntoji, N. and Kuppast, V. (2017) Study of Aircraft Wing with Emphasis on Vibration Characteristics. International Journal of Engineering Research and Applications, 7, 1-8.

[3] Kavya, G. and Reddy, B.C.R. (2015) Design and Finite Element Analysis of Aircraft Wing Using Ribs and Spars. International Journal \& Magazine of Engineering, Technology, Management and Research, 2, 1442-1455. 
[4] Zhu, L. and Childs, P.R.N. (2018) Light-Weighting in Aerospace Component and System Design. Propulsion and Power Research, 7, 103-119.

https://doi.org/10.1016/j.jppr.2018.04.001

[5] Inman, D.L. (2014) Engineering Vibration. 4th Edition, Prentice Hall, Upper Saddle River.

[6] Airfoil Tools. http://www.airfoiltools.com/airfoil/details?airfoil=ah79100a-il 